\title{
Good Laboratory Practice Indicator Flag
}

National Cancer Institute

\section{Source}

National Cancer Institute. Good Laboratory Practice Indicator Flag. NCI Thesaurus. Code C120944.

An indicator that can be set or unset in order to signal whether the study has been conducted using good laboratory practice standards. 